\title{
Application of Multiple Imaging Tools for Organic Material Characterization in Shale Reservoirs
}

\author{
Zhengfan Liu ${ }^{1}$, Lori Hathon ${ }^{1}$ and Michael T. Myers ${ }^{1}$ \\ ${ }^{1 .}$ Department of Petroleum Engineering, University of Houston, Houston, TX, USA
}

Vitrinite reflectance and Rock-Eval pyrolysis (Tmax) are the two standard techniques applied to determine source rock maturity. For samples with small volumes of organic material (OM), or for high maturity samples, or where vitrinite is rare to absent (e.g. pre-Silurian rocks which do not have vitrinite macerals), the application of these techniques is difficult. Additionally, in organic rich source rocks vitrinite is rare, making reflectance analysis non-robust. Because of these difficulties, there is a need for new methods to accurately characterize of shale kerogen thermal maturity.

In SEM (scanning electron microscopy) imaging, different OM types in shale reservoirs are identified based on their morphology and distribution, as well as gray level contrast. However, for definitive OM type determination, this information is often not enough. There is a need to link the distribution and morphology of the organic matter types with other information. Raman spectroscopy, based on molecular vibrations, can provide information on chemical bonds in individual organic components [1].

Compared to vitrinite reflectance and Rock-Eval pyrolysis, Raman spectroscopy has several advantages. First, the laser focal volume is around $3 \mu \mathrm{m}^{3}$ under the Raman microscope, enabling the analysis of small volumes of dispersed OM. Second, the technique detects the Raman spectrum of target samples based on their chemical composition instead of their optical reflectance. The Raman spectrum is not influenced by optical bireflectance or heterogeneity. Third, Raman techniques can also be applied to samples with no vitrinite macerals.

A relationship is demonstrated between Raman spectra and thermal maturity of coal and kerogen samples [2]. We intend to develop similar relationships for the various OM types in unconventional reservoirs. We simultaneously use a high resolution (50 micron) acoustic microscope to directly measure velocities associated with varying organic matter content in highly laminated samples.

We performed Raman spectroscopic analysis and SEM imaging on OM rich shales at different thermal maturity levels. Shale kerogens with low thermal maturity typically exhibit a strong fluorescence background that can mask the Raman spectrum. By selection of the appropriate Raman excitation laser, we have successfully measured the Raman spectrum of low maturity ( $0.5 \%$ Ro) OM. The Raman spectrum of shale kerogen shows a D band around $1350 \mathrm{~cm}^{-1}$ and a $\mathrm{G}$ band around $1590 \mathrm{~cm}^{-1}$. Several associated bands are also revealed by deconvolution. We are able to pinpoint different OM types in the field of view by simultaneously viewing the sample in reflected light and obtaining a Raman spectrum. Different OM types are shown to have different Raman spectra. As shown in Fig. 1, Raman spectrum of terrestrial OM shows a much lower trough between the two peaks compared to that of marine OM and bitumen. Fig. 2 shows that marine OM spectra show D4 peak center above $1263 \mathrm{~cm}^{-1}$, which is higher than that of terrestrial and bitumen. Thermal maturity of the shale samples are correlated with the Raman spectrum for both marine and terrestrial sedimentary OM, as well as bitumen. Our results are shown together with SEM imaging of OM of various types (e.g. bitumen, marine Type II kerogen, and terrestrial OM) so that an integrated understanding of the Raman spectra, OM type, and maturity is achieved. 
The acoustic microscope is used to measure the acoustic properties of the macerals (where they are large enough) and the acoustic properties of different lamina in the sample that vary in mineralogy, porosity, and TOC. From detailed SEM imaging and image analysis, we are able to correlate acoustic properties with TOC and the changing dynamic modulus of the OM with increasing thermal maturity. This study demonstrates the feasibility of Raman spectroscopy as a thermal maturity indicator for shale kerogen, and as a tool for identifying different OM types.

References:

[1] Raman C V, Krishnan K S., Nature 121 (1928), p. 501.

[2] Kelemen, S. R. and H. L., Fang Energy \& Fuels 15(3) (2001), p. 653.
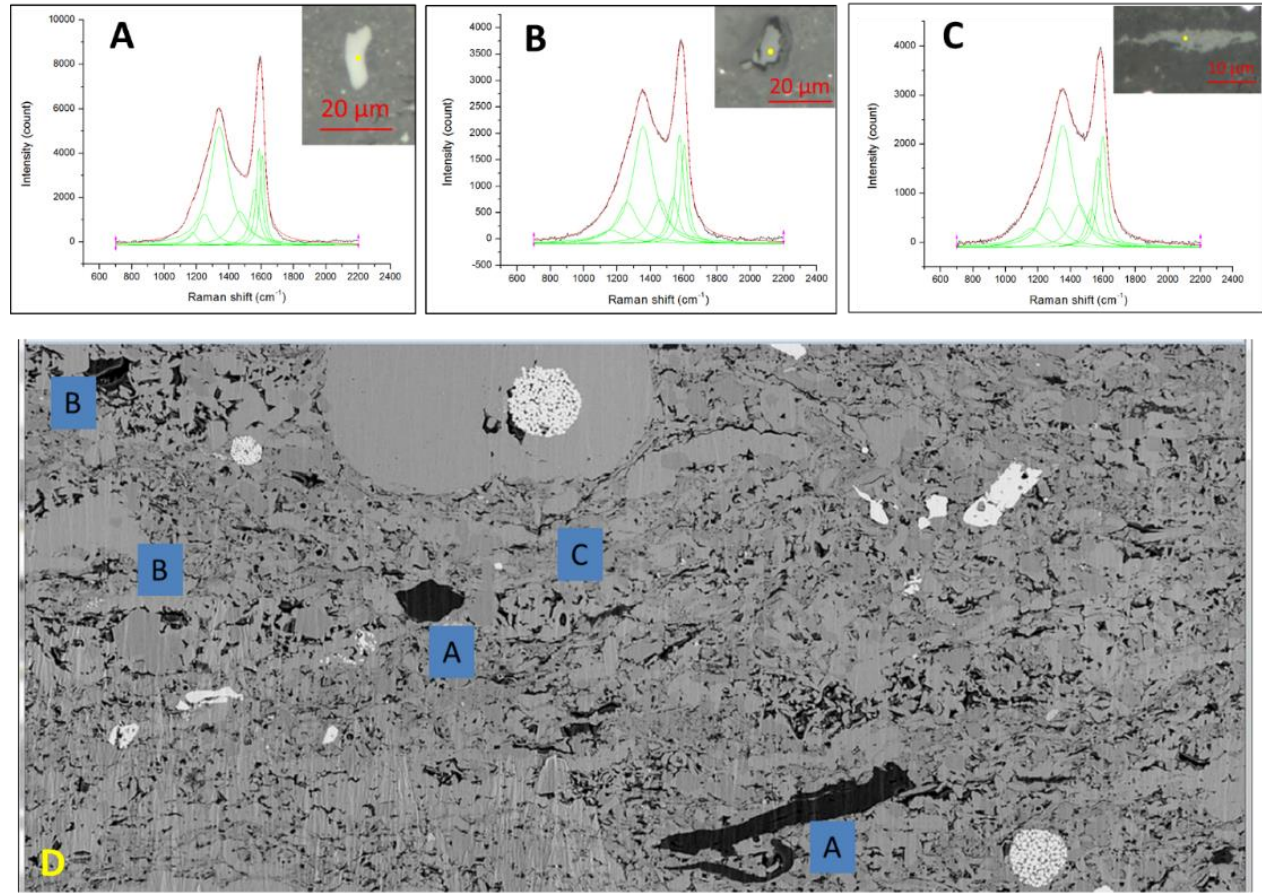

Figure 1. Different organic material types in a $0.9 \%$ Ro shale sample observed with Raman spectroscopy and SEM. A) Raman spectrum of terrestrial organic material. B) Raman spectrum of bitumen. C) Raman spectrum of marine organic material. D) SEM image showing different organic material type.

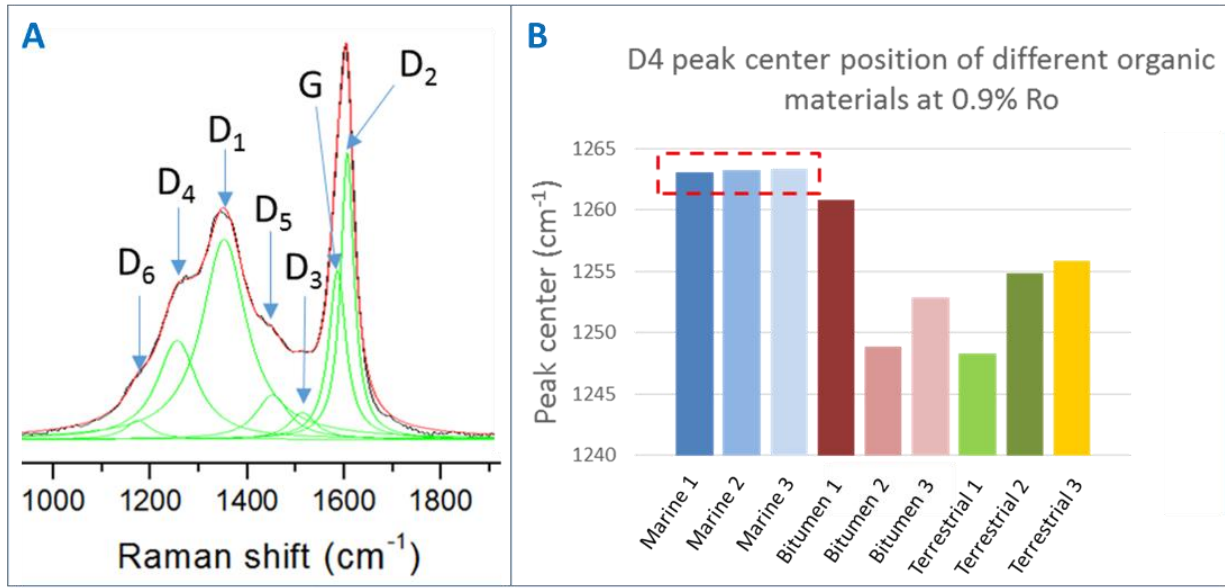

Figure 2. A) Band deconvolution of Raman spectrum of a marine OM at 1.2\%Ro maturity. B) D4 peak center position of different organic material types. For marine, that value is above $1263 \mathrm{~cm}^{-1}$. 